PAPER

\title{
Tuberculous meningitis in BCG vaccinated and unvaccinated children
}

\author{
R Kumar, A Dwivedi, P Kumar, N Kohli
}

See Editorial Commentary, p 1470

J Neurol Neurosurg Psychiatry 2005;76:1550-1554. doi: 10.1136/jnnp.2005.065201

Background: A modified clinical presentation of tuberculous meningitis (TBM) in children vaccinated with BCG has been described in the literature. However, most reports are old and not based on actual comparisons and tests of significance. Also, neuroimaging features were not compared. With large scale BCG coverage, it becomes pertinent to describe the "modified" presentation and identify any significant differences between vaccinated and unvaccinated children with TBM.

See end of article for authors' affiliations

Methods: A total of 150 consecutive hospitalised children (96 unvaccinated, 54 vaccinated) were enrolled. They all satisfied predefined criteria for diagnosis of TBM. Clinical and radiological features of children with/without a BCG scar were compared.

Correspondence to: Professor R Kumar, HIG

Results: Univariate analysis revealed that the vaccinated children with TBM had significantly lower rates of 111, Sec E, Aligani, altered sensorium (68.5\% v 85.4\% unvaccinated; OR $2.2(1.1$ to 6.2$) ; p=0.019)$ and focal neurological deficits $(20.3 \%$ v 39.5\% unvaccinated; OR 2.6 (1.1 to 6.0); $p=0.016)$, and higher mean (SD) Glasgow Coma Scale score (10.2 (3.4) v 8.76 (2.7) unvaccinated; $p=0.010$ ) and cerebrospinal fluid cell count Lucknow (UP), India sancharnet.in (210.9 v 140.9 unvaccinated; $p=0.019)$. No significant radiological differences were seen. Short term outcome was significantly better in the vaccinated group with $70 \%$ of the total severe sequelae and $75 \%$ of

Received 10February 2005 Revised version received the total deaths occurring in the unvaccinated group $(p=0.018)$.

27 May 2005

Accepted 27 May 2005

Conclusion: Children with TBM who have been vaccinated with BCG appear to maintain better mentation and have a superior outcome. This may in part be explained by the better immune response to infection, as reflected in the higher CSF cell counts in this group in the present study.

$\mathrm{T}$ aberculosis continues to be a major public health problem globally. India is a major contributor to this global burden, harbouring nearly a third of all cases. ${ }^{1}$ Tuberculous meningitis (TBM) is the most dangerous form of tuberculosis and is, in particular, seen in children. TBM remains an important cause of hospitalisation, death, and permanent neurological disability in children in India. Over the years there has been no noteworthy decline in the incidence of this deadly disease, despite the claims of high vaccination coverage with the bacille Calmette Guérin (BCG) vaccine. ${ }^{23}$ Although the BCG vaccine has been used for over 80 years, there remains a shadow of doubt regarding its value in protection against tuberculosis. ${ }^{45}$ In large community trials the protective efficacy of BCG varies from $0 \%$ to $80 \%{ }^{6-9}$ The BCG vaccine trial in the Chingleput district in southern India showed no protective effect of the vaccine against adult pulmonary tuberculosis. ${ }^{10}$

Although the protective efficacy of BCG vaccination in adult forms of the disease is doubtful, it is held to be useful in preventing the spread of and improving outcome of tubercular infection. ${ }^{11-13}$ Vaccinated children mobilise their cellular immune responses more effectively compared with unvaccinated children when exposed to natural tuberculous infection, thereby experiencing fewer haematogenous complications such as miliary tuberculosis and TBM. However, even this protection is only relative and may be overcome in presence of a heavy infecting dose from a household contact, in the presence of severe malnutrition, and because of waning immunity many years after vaccination. ${ }^{14-16}$

There are few reports in the literature that describe a distinctive clinical presentation of TBM in BCG vaccinated children. ${ }^{17-22}$ These reports are not based on actual comparisons and statistical differences between vaccinated and unvaccinated children and were mostly anecdotal in nature. Also, most of these studies were conducted in the

pre-computed tomography (CT) era and radiological features were not reported.

It is important to recognise the full clinical spectrum of TBM in BCG vaccinated children so that the diagnosis is not delayed. With more children being vaccinated nowadays, the clinical spectrum of TBM may be changing. We therefore undertook this prospective study to compare the clinical and radiological features of TBM in BCG vaccinated and unvaccinated children.

\section{PATIENTS AND METHODS}

We conducted this cross-sectional study over a period of two years, from July 2002 to July 2004, in the pediatric wards of King George Medical University Hospital, Lucknow, India. We included those children between one month and 12 years of age, who were admitted to the hospital and satisfied certain predefined criteria for diagnosis of TBM, provided that they had not received antitubercular treatment in the last three months. The diagnostic criteria for TBM were:

- clinical presentation with fever of 14 days or more along with any neurological manifestation and a progressive course

- cranial CT scan showing two or more of the following:

- hydrocephalus

- enhancement in basal cisterns or sylvian fissures

- tuberculoma

- cerebrospinal fluid (CSF) pleocytosis $\left(>10\right.$ cells $\left./ \mathrm{mm}^{3}\right)$.

Abbreviations: BCG, bacille Calmette Guérin (vaccine); CSF, cerebrospinal fluid; TBM, tubercular meningitis 
All enrolled children underwent a work-up in accordance with a predesigned protocol including a detailed history taking, physical examination, investigations, and follow up. We assessed the children's nutritional status by weight for age percentage using standards derived from Indian children, ${ }^{23}$ and recorded the presence/absence of a BCG scar on the left shoulder. The sensorium was assessed using the Glasgow Coma Scale modified for children. Bradycardia with hypertension and/or an abnormal breathing pattern were considered as features of raised intracranial tension.

The investigations carried out included complete blood count, tuberculin test with 5TU PPD (tuberculin purified protein derivative), chest $x$ ray, CSF examination for cells, protein, and sugar, bacterial and mycobacterial cultures, and a cranial contrast-enhanced CT scan. The latter was usually done within 72 hours of admission with a third generation scanner, and 10-12 axial cuts were taken. We measured ventricular size and graded the hydrocephalus as mild, moderate, or severe according to Meese et al. ${ }^{24}$ Basal enhancement was also graded as mild, moderate, or severe according to Bhargava et al. ${ }^{25}$ Hydrocephalus was classified as communicating if the fourth ventricle was also dilated and obstructive if the fourth ventricle was normal.

We followed the hospital's protocol with regard to therapy. All the patients were started on a four drug antitubercular treatment along with steroids. The antitubercular regimen consisted of:

- intramuscular injection of streptomycin $30 \mathrm{mg} / \mathrm{kg}$ per day for two months

- pyrazinamide (tablet) $30 \mathrm{mg} / \mathrm{kg}$ per day for two months

- rifampicin (tablet) $10 \mathrm{mg} / \mathrm{kg}$ per day for 12 months

- isoniazid (tablet) $5 \mathrm{mg} / \mathrm{kg}$ per day for 12 months.

Steroids were given in the form of oral prednisolone $1-2 \mathrm{mg} /$ $\mathrm{kg}$ per day for four to six weeks and tapered off.

As all of the children were hospitalised, we could check compliance daily by questioning their attendants. We recorded the daily progress in hospital. Outcome at discharge was classified as follows:

- normal

- mild sequelae, such as isolated focal neurological deficit, but ambulatory and able to perform daily activities

- severe sequelae, such as frank mental regression, frank motor deficits leading to inability to perform routine activities, blindness, deafness, or vegetative state

- death.

\section{Statistical analysis}

We entered data in a Microsoft Excel worksheet and used SPSSwin software for data analysis. The clinical features, results of the investigations, and outcome of the children with and without a BCG scar were compared by univariate analysis; two sample $t$ tests were used for continuous variables and $\chi^{2}$ tests for nominal and ordinal variables to identify features associated with BCG vaccination. The sample size was calculated for difference between proportions. With $\alpha=0.05, \beta=0.2$, and smaller proportion $=0.2$, and to detect a difference of $20 \%$ between the two groups, the sample size was calculated to be 54 per group. ${ }^{26}$

\section{Ethical approval}

We did not obtain formal ethical approval because the study was purely observational and patients' identities were not revealed. However, informed verbal consent was obtained from the guardian of each child.

\section{RESULTS}

A total of 150 children with TBM were enrolled in the study, of whom 54 had a BCG scar. Tables 1-4 provide comparisons of the symptoms, signs, investigative findings, and outcomes between BCG vaccinated and unvaccinated children. Of the 10 demographic/historical features compared in table 1, only altered sensorium was significantly different in the two groups, being higher in the non-vaccinated group. Of the 13 clinical signs shown in table 2, the Glasgow Coma Scale at admission was significantly lower and prevalence of neurological deficits was significantly higher in the unvaccinated group. Comparison of the investigative findings (table 3) revealed significant differences between the groups only in the CSF cell count, which was higher in the vaccinated group. Finally, outcome was significantly better in the vaccinated group (table 4 ).

\section{DISCUSSION}

A number of workers have remarked on the modified pattern of TBM in BCG vaccinated children but most observations have been anecdotal in nature. To date there are only a few studies in the world literature that have compared clinical features of BCG vaccinated and unvaccinated children with TBM. $^{20}{ }^{21}$ Further, there is scant literature comparing the radiological (neuroimaging) features in these two groups of children. ${ }^{20}$ As more and more children are being vaccinated with BCG, the clinical spectrum of the disease (TBM) may be changing. It is important to document the clinical and radiological differences so as to recognise the full spectrum of the disease.

\begin{tabular}{|c|c|c|c|c|}
\hline & \multicolumn{2}{|l|}{ BCG scar } & \multirow[b]{2}{*}{ OR $(95 \% \mathrm{Cl})$} & \multirow[b]{2}{*}{$p$ value } \\
\hline & Absent & Present & & \\
\hline No of children & 96 & 54 & & \\
\hline Age (in months) $\dagger$ & $45.63(37.5)$ & $46.5(36.1)$ & & 0.754 \\
\hline Duration of illness (days) $\dagger$ & $45.2(52.3)$ & $35.1(38.3)$ & & 0.157 \\
\hline Tonic spasms $\ddagger$ & $44(45.8)$ & $23(42.5)$ & $1.1(0.5$ to 2.4$)$ & 0.701 \\
\hline Generalised clonic seizuresł & $38(39.5)$ & $16(29.6)$ & $1.6(0.7$ to 3.4$)$ & 0.272 \\
\hline Partial seizures $¥$ & $11(11.4)$ & $4(7.4)$ & $2.7(0.7$ to 10.8$)$ & 0.097 \\
\hline Convulsion frequencyt per day & $5.19(4.9)$ & $3.8(4.7)$ & & 0.485 \\
\hline Headacheł & $46(47.9)$ & $29(53.7)$ & $0.9(0.4$ to 2.2$)$ & 0.899 \\
\hline Vomiting $\ddagger$ & $72(75.0)$ & $41(75.9)$ & $0.8(0.4$ to 1.8$)$ & 0.670 \\
\hline Altered sensorium $\ddagger$ & $82(85.4)$ & $37(68.5)$ & $2.2(1.1$ to 6.2$)$ & $0.019^{*}$ \\
\hline Contact history $\ddagger$ & $45(46.8)$ & $32(59.2)$ & $0.6(0.3$ to 1.3$)$ & 0.145 \\
\hline
\end{tabular}


Table 2 Comparison of clinical signs in vaccinated and unvaccinated children

\begin{tabular}{|c|c|c|c|c|}
\hline & \multicolumn{2}{|l|}{ BCG scar } & \multirow[b]{2}{*}{ OR $(95 \% \mathrm{Cl})$} & \multirow[b]{2}{*}{ p value } \\
\hline & Absent & Present & & \\
\hline No of children & 96 & 54 & & \\
\hline Weight for age \%† & $67.9(13.8)$ & $70.5(12.8)$ & & 0.470 \\
\hline Glasgow Coma Scale score† & $8.76(2.7)$ & $10.2(3.4)$ & & $0.010^{*}$ \\
\hline Fundus blurring/papilloedema & $40(41.6)$ & $21(38.8)$ & $1.1(0.5$ to 2.3$)$ & 0.739 \\
\hline Fundus pallor/atrophy & $22(22.9)$ & $14(25.9)$ & $0.8(0.4$ to 2.0$)$ & 0.678 \\
\hline Meningeal signs $\ddagger$ & $64(66.6)$ & $35(64.8)$ & $1.1(0.5$ to 2.2$)$ & 0.818 \\
\hline Focal deficits $\ddagger$ & $38(39.5)$ & $11(20.3)$ & 2. $6(1.1$ to 6.0$)$ & $0.016^{*}$ \\
\hline Cranial nerve palsył & $40(41.7)$ & $18(33.3)$ & $1.4(0.7$ to 2.8$)$ & 0.353 \\
\hline Generalised $\uparrow$ tone‡ & $68(70.6)$ & $32(59.2)$ & $1.7(0.8$ to 3.6$)$ & 0.148 \\
\hline Clonusł & $20(20.8)$ & $17(31.4)$ & $0.6(0.2$ to 1.3$)$ & 0.146 \\
\hline Plantar extensor $\ddagger$ & $81(84.3)$ & $40(74.0)$ & $2.0(0.8$ to 5.1$)$ & 0.087 \\
\hline Abnormal movements $\ddagger$ & $30(31.2)$ & $12(22.2)$ & $1.6(0.7$ to 3.7$)$ & 0.237 \\
\hline Decerebration $\ddagger$ & $25(26.0)$ & $11(20.3)$ & $1.4(0.6$ to 3.3$)$ & 0.435 \\
\hline Signs of $\uparrow$ intracranial tension $\ddagger$ & $42(43.7)$ & $16(29.6)$ & $1.8(0.9$ to 4.0$)$ & 0.088 \\
\hline
\end{tabular}

The strengths of the present study include the prospective enrolment of children and careful documentation using a standardised predesigned data collection form. The case definition of TBM was also predefined. These TBM criteria are used by paediatricians throughout India. Except for fungal meningitis, which admittedly is a rare entity, other meningoencephalitides are unlikely to be misclassified as TBM by these criteria. The Indian Academy of Pediatrics Working Group on Tuberculosis has laid special emphasis on cranial CT scan for diagnosis of TBM. ${ }^{27}$ We deliberately kept our diagnostic criteria sufficiently broad so that we could include as wide a spectrum of the disease as possible. Although diagnosis based on CSF culture for mycobacteria or on polymerase chain reaction for mycobacterial DNA in CSF yield the widest possible range of disease, these investigations were not usually available to us and the results would have been available been only after several weeks. Weight for age was adopted as the criterion for grading malnutrition. Other parameters such as skinfold thickness or mid-arm circumference may have added to the measure of nutritional status, but weight for age is the single most important parameter that is widely used in assessment of nutritional status and grading of malnutrition. A BCG scar was considered as the marker for vaccination. In India, patients seldom keep a record of vaccinations given, and thus the BCG scar is the most practical method of ascertaining prior vaccination with BCG. However, scars could have faded in a small proportion of patients. We assessed short term outcome only even though we often see the neurological status of patients with TBM improve over months or years, and therefore some of our poor outcomes may change for the better with time. However, in the present study patients with good outcome would at least have been correctly classified.

Table 3 Comparison of investigative findings in vaccinated and unvaccinated children

\begin{tabular}{|c|c|c|c|c|}
\hline & \multicolumn{2}{|l|}{ BCG scar } & \multirow[b]{2}{*}{ OR $(95 \% \mathrm{Cl})$} & \multirow[b]{2}{*}{$\mathrm{p}$ value } \\
\hline & Absent & Present & & \\
\hline No of children & 96 & 54 & & \\
\hline \multicolumn{5}{|l|}{ Cerebrospinal fluid } \\
\hline Cellst & $140.9(156.6)$ & $210.9(288.4)$ & - & $0.019^{*}$ \\
\hline Polymorphonuclear leucocytes \%† & $35.4(31.4)$ & $32.7(33.0)$ & - & 0.431 \\
\hline Proteint & $134.3(143.9)$ & $135.1(189.7)$ & - & 0.403 \\
\hline Sugart & $37.3(20.9)$ & $38.3(21.4)$ & - & 0.306 \\
\hline$X$ ray chest (miliary or consolidation) $\ddagger$ & $26(27.0)$ & $14(25.9)$ & $1.0(0.4$ to 2.4$)$ & 0.923 \\
\hline \multicolumn{5}{|l|}{ Computed tomography scan } \\
\hline Hydrocephalus $\ddagger$ & 94 (97.9) & $54(100)$ & $0.0(0.0$ to 7.3$)$ & 0.285 \\
\hline Communicating $\ddagger$ & $44(45.8)$ & $16(29.6)$ & $2.0(0.9$ to 4.3$)$ & 0.051 \\
\hline Obstructiveł & $44(45.8)$ & $31(57.4)$ & $0.6(0.3$ to 1.3$)$ & 0.173 \\
\hline Basal enhancementł & $81(84.3)$ & $44(81.4)$ & $1.2(0.5$ to 3.2$)$ & 0.648 \\
\hline \multicolumn{5}{|l|}{ Degree of enhancement: } \\
\hline 1 & 4 & 1 & - & 0.425 \\
\hline 2 & 15 & 5 & & \\
\hline 3 & 30 & 17 & & \\
\hline 4 & 3 & 1 & & \\
\hline Periventricular leak & 70 (72.9) & $37(68.5)$ & $1.2(0.6$ to 2.7$)$ & 0.567 \\
\hline Tuberculomał & $8(8.3)$ & $4(7.4)$ & $1.1(0.3$ to 4.8$)$ & 0.840 \\
\hline Infarctł & $17(17.7)$ & $9(16.6)$ & $1.1(0.4$ to 2.9$)$ & 0.871 \\
\hline \multicolumn{5}{|l|}{ Ventricular size } \\
\hline 0 & 1 & 1 & - & 0.457 \\
\hline 1 & 11 & 5 & & \\
\hline 2 & 32 & 15 & & \\
\hline 3 & 16 & 5 & & \\
\hline \multicolumn{5}{|c|}{$\begin{array}{l}\text { *Significant. } \\
+ \text { Values are mean (SD). A two sample } t \text { test was used for comparison of means. } \\
\ddagger \text { Values are } n(\%) . \chi^{2} \text { tests were used for comparison of proportions. }\end{array}$} \\
\hline
\end{tabular}


Table 4 Comparison of outcomes of vaccinated and unvaccinated children with TBM

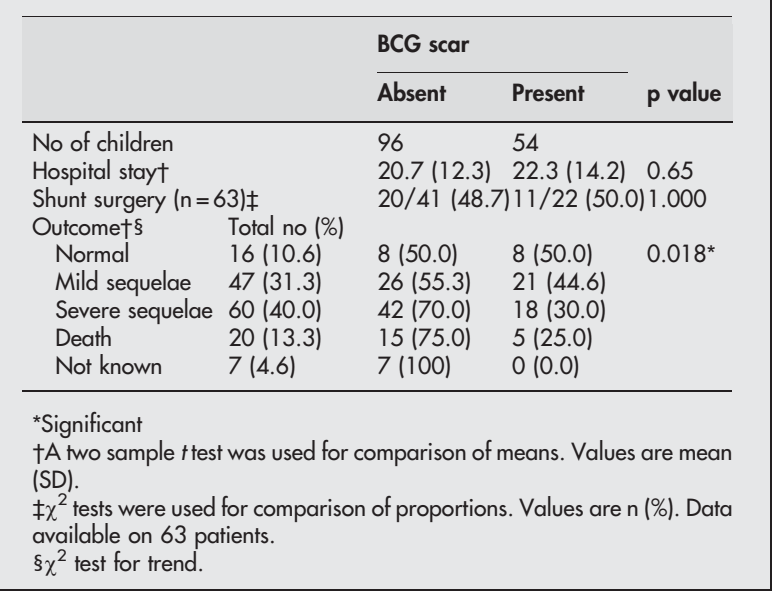

Our statistical comparisons of the two groups included 38 variables, of which five showed significant differences. It can be argued that the few significant differences could have occurred by chance. However, it must be taken into account that the significant differences were found among related variables from the history and physical examination-this shows a true association. We did not adjust the level of significance. In fact, if the cut-off for the $\mathrm{p}$ value had been lowered to 0.01 or 0.005 , none of the differences would have been significant.

About two thirds of children with TBM in the present study were unvaccinated. To enrol 54 vaccinated children with TBM, we therefore had to enrol 96 unvaccinated children. Our hospital caters in particular to the poor and seriously sick children of the city of Lucknow and its surrounding areas. Therefore, it is likely that we see a large proportion of the individuals contracting TBM in this area especially when the disease reaches an advanced stage. Whether this reflects the true vaccination coverage in the community is, however, doubtful. Since this was a hospital based study, it is possible that unvaccinated children, who have a higher risk of getting TBM, are seen more frequently by us.

The comparison of clinical history in the vaccinated and unvaccinated groups revealed a significantly higher proportion with altered sensorium in the unvaccinated group. Table 1 shows that the average duration of symptoms at admission was 10 days longer in the vaccinated group. The difference was not statistically significant. Unvaccinated children likely come from lower socioeconomic backgrounds and uneducated families who are less aware of or simply not able to recognise or report their child's symptoms early on. On examination the Glasgow Coma Scale score was significantly lower in this group. In a study of 80 cases between 1973 and 1975, Udani et al observed that the "conscious" type of TBM was three times commoner in vaccinated children. ${ }^{22}$ They also found that localised forms of TBM occurred more commonly in BCG vaccinated children, whereas "classic" TBM was seen twice as often in the unvaccinated children studied. Various brain stem syndromes due to localised brain involvement have been described in vaccinated children by these pioneers in the field. ${ }^{21}$ Although we did not observe such brain stem syndromes or cranial nerve palsies more commonly in our vaccinated group, our study supports Udani et al's observations that BCG vaccinated children have a significantly higher rate of conscious-type TBM. We also found significantly higher rate of focal neurological deficits in the unvaccinated group.
The only investigation that was significantly different between the groups was the mean CSF cell count, which was higher in the vaccinated group. This may reflect a better immune response and cellular reaction to the infection. However, Udani et al reported a higher rate of "serous" TBM (51.3\% v $18.7 \%$ in unvaccinated) in their series. ${ }^{21}$ We did not find any instances of serous TBM in the present study because one of our criteria for diagnosis was CSF pleocytosis. The radiological findings of the two groups were not significantly different. Although communicating hydrocephalus was seen in $45.8 \%$ of the unvaccinated children in contrast with $29.6 \%$ in vaccinated group, this difference just failed to reach statistical significance.

Finally, our study indicates that outcome of TBM was better in the vaccinated children; however, we studied the short term outcome only. This has been reported by earlier workers also. Guller et al (1998) studied the effect of neonatal BCG vaccination on clinical and laboratory profiles of and mortality among children with TBM in Turkey. ${ }^{20}$ Although the rate of family contact of tuberculosis, age distribution, clinical features, and laboratory investigations were not significantly different between the vaccinated and unvaccinated children, mortality was only a third in the vaccinated group. Udani also stated that one of the most important factors affecting prognosis in TBM is BCG vaccination, and found that death rate due to miliary tuberculosis and TBM was twice as high in unvaccinated children. ${ }^{122}$ BCG vaccination may be one of the factors influencing the outcome of TBM. Other factors that might play a role include age, stage of disease at diagnosis, immune and nutritional status, household contact, compliance and coexistence of other illnesses. Of these, the latter four may be influenced by socioeconomic factors and living conditions. We did not evaluate the socioeconomic status of our patients but data on nutritional status and household contact were collected and compliance was ensured because the patients were hospitalised. Although this univariate analysis suggests an association between BCG vaccination status and better outcome in TBM, it would be interesting to undertake a multivariate analysis of factors influencing outcome of TBM to see if this association holds true even after controlling for the effect of other variables.

\section{CONCLUSION}

Although BCG vaccination does not totally prevent occurrence of TBM our results support earlier studies suggesting that children who have been vaccinated with BCG appear to maintain better mentation and ultimately have a better outcome than unvaccinated children. Our study did not reveal any significant differences in neuroradiological features to explain this finding. The better outcome may in part be explained by the better mobilisation of cell mediated immune response to infection as is reflected in the higher mean CSF cell count in vaccinated children in our study.

\section{Authors' affiliations \\ R Kumar, A Dwivedi, P Kumar, Department of Pediatrics, King George Medical University, Lucknow, India \\ N Kohli, Department of Radiodiagnosis, King George Medical \\ University, Lucknow, India \\ Competing interests: none declared}

\section{REFERENCES}

1 World Health Organization. Joint Tuberculosis Program Review, India, February 2000. New Delhi: WHO Regional Office for South East Asia.

2 Rao SP, Bharambe MS. Vaccine preventable diseases in Eastern Maharashtra: a hospital based analysis. Indian Pediatr 1991;28:629-33.

3 Somu N, Vijayasekaran D, Rajkumar T, et al. Tuberculous disease in a pediatric referral center -16 years experience. Indian Pediatr 1994;31:1245-9 
4 Raju VB, Narmada R. Evaluation of BCG vaccination in children below 6 years. Indian Pediatr 1970;7:532-41.

5 Deshpande NS, Deshpande SV. Tuberculosis in BCG vaccinated children. Indian Pediatr 1995;32:676-78.

6 Rosenthal SR, Lewinson E, Liverght D, et al. BCG vaccination against tuberculosis in Chicago: a 20 year study statistically analysed. Pediatr 1961;28:522-41.

7 Comstock GW, Webster RG. Tuberculosis studies in Muscogee County, Georgia: A 20 year evaluation of BCG vaccination in a school population. Am Rev Respir Dis 1969;100:839-45.

8 Comstock GW, Livesay VT, Woolpart SF. Evaluation of BCG vaccination among Puerto Rican children. Am J Public Health 1974:64:283-91.

9 Hart PD, Sutherland I. BCG and vole bacillus vaccines in the prevention of tuberculosis in adolescence and early adult life. Final report of the Medical Research Council. BMJ 1997;2:293-5.

10 Datta M, Vallishayee RS, Diwakara AM. Tuberculosis Research Centre (ICMR), Chennai: 15 year follow up trial of BCG vaccine in South India for tuberculosis prevention. Indian J Med Res 1999;110:56-9.

11 Chandra $P$. Tuberculosis in BCG vaccinated and unvaccinated children. Indian Pediatr 1975; 12:424-9.

12 Udani PM. Protective value of BCG vaccination. Indian Pediatr 1982;14:739-52.

13 Costa MC, Mota EL, Pinto LL. Protective effect of intradermal BCG on tuberculous meningitis. Bol Oficina Sanit Panam 1991;110:26-32.

14 Parthasarathy A, Narmada R, Krishnamoorthy KA. Tuberculosis in BCG vaccinated children. Indian Pediatr 1982;19:785-91.

15 Bhandari B, Mandowara SL. A study of tuberculosis in BCG vaccinated children. Indian Pediatr 1982;19:865-8.
16 Kumar $\mathbf{P}$, Kumar $\mathrm{R}$, Srivastava KL, et al. Protective role of $\mathrm{BCG}$ vaccination against tuberculous meningitis in Indian children: A reappraisal. Natl Med J India 2005; 18:7-11.

17 Paul MF. Tuberculosis in BCG vaccinated children in Singapore. Arch Dis Child 1961;36:530-6.

18 Tandon PN, Singh B, Mahapatra LN, et al. Experimental tuberculosis of central nervous system. Neurol India 1970;18:81-5.

19 Weigel B. Changes in the tuberculosis of the central nervous system since the introduction of prophylactic BCG vaccination and tuberculostatic therapy. Zentralbl Allg Pathol 1976;120:21-3.

20 Guller N, Ores U, Somer A, et al. The effect of prior BCG vaccination on clinical and radiographic presentation of tuberculous meningitis in children in Istanbul, turkey. Int J Tubercul Lung Dis 1998;2:885-90.

21 Udani PM, Bhave SY, Tilak AM, et al. CNS tuberculosis with clinical picture modified by BCG vaccination and/or drug therapy. I Jj Group Hosp Grant Med Coll 1979;24:3.

22 Udani PM, Parekh UC, Dasfur DK. Some neurological syndromes in CNS tuberculosis. Neurol India 1972; Suppl:63-9.

23 Agarwal DK, Agarwal KN, Upadhyaya SK, et al. Physical and sexual growth of affluent Indian children. Indian Pediatr 1992;29:1203-84.

24 Meese W, Lanksch W, Wende S. Diagnosis and post operative follow up studies of infantile hydrocephalus using computerised tomography. In: Lanksch W, Kazner E, eds. Cranial Computerised Tomography. Berlin: Spinger-Verlag, 1976

25 Bhargava S, Gupta AK, Tandon PN. Tuberculous meningitis-a CT study. Br J Radiol 1982;55:19-96.

26 Hulley SB, Cummings SR. Designing Clinical Research: An epidemiologic Approach. Baltimore: William \& Wilkins, 1985:216.

27 IAP Working Group on Childhood Tuberculosis. Consensus Statement on diagnosis of childhood tuberculosis. Indian Pediatr 2004;41:146-58. 